\title{
NOVEL DESIGN AND EXPERIMENTAL VALIDATION OF A CONTRACTION NOZZLE FOR AERODYNAMIC MEASUREMENTS IN A SUBSONIC WIND TUNNEL
}

\author{
María Rodríguez Lastra, Jesús Manuel Fernández Oro, Mónica Galdo Vega, Eduardo Blanco Marigorta, \\ Carlos Santolaria Morros
}

\author{
Área de Mecánica de Fluidos. Universidad de Oviedo, \\ Gijón, (Asturias) Spain 33271 \\ Email: rodriguezlmaria.uo@uniovi.es
}

\begin{abstract}
A novel design for a contraction nozzle, based on a logarithmic profile, is presented and developed to enhance aerodynamic measurements in a low-speed wind tunnel. The improvements obtained with this new proposal are validated, both, numerical and experimentally. As a starting point, four different wind tunnel contraction profiles are firstly considered and tested using the Computational Fluid Dynamics (CFD) package ANSYS FLUENT ${ }^{\circledR}$. Both polynomial, due to its classical inclusion for wind tunnels, and logarithmic profiles, due to its expected enhancement, have been studied in terms of avoidance of separation of the boundary layer, procurement of a maximum level of exit-flow uniformity, and minimum turbulence levels at the outlet. Numerical comparison between obtained results shows the benefits of the new logarithmic profile developed by the authors, which was finally employed to construct the nozzle. To characterize its real performance, intensive experimental measurements have been conducted using pressure transducers and both single and dual hot wire anemometry. The pressure coefficient along the nozzle sidewall reveals an optimal evolution, matching perfectly with the theoretical design. In addition, low levels of turbulence and high flow uniformity is confirmed at the nozzle discharge. Turbulence intensities below $0.7 \%$ are obtained for the whole range of velocities available in the wind tunnel, and even practically constant uniform flow is obtained for all the traverses tested for validation. These indicators conclude that the contraction designed with the new profile is a good enhancing of the wind tunnel benefits.
\end{abstract}

\section{INTRODUCTION}

Wind tunnels represent an essential tool for fluid flow research and model testing. They allow to provide significant data about flow characteristics around immersed bodies and aeroacoustic noise, when the test section is also conditioned to perform acoustic measurements. In the context of modern designs of wind tunnels, the Fluid Dynamics group of the University of Oviedo has developed its own facility in 2008 integrating, both, aerodynamic and aeroacoustic experiments. This facility is provided with a characteristic open section of 1 $\mathrm{m}^{2}$ and anechoic paneling to increase its experimental capabilities. A number of constructive modifications have been performed since then to improve current measurements, including the design and build of an optimized contraction that could increase the quality of the air flow in the test section.

The design of the optimal contraction section in a wind tunnel is critical to obtain valuable data from the facility. A good choice is a crucial milestone to assure high-quality flow characteristics and, therefore, it deserves special attention. Typically, converging nozzles have been constructed using a pair of cubic polynomials where, for a fixed length and contraction ratio, the location of the joining point has been found to be determinant in the optimization of the designs [14]. Years ago, the analytical resolution of inviscid flow equations to study the flow field, required a lot of time. For that reason, design charts for nozzles were developed, and recurrent calculations were avoided [1-6]. However, due to the great computational advances and the development of more efficient codes, CFD has currently become an essential tool to optimize wind tunnel contractions. It is an accurate way to analyze the flow behavior within the nozzle and, moreover, allows geometric parameters to be optimized without relatively increasing costs of computation. Because of the smooth transition of the side walls and the high Reynolds number reached within the nozzle, boundary layer growth is controlled and viscous computations are expected to be reasonably simple (no swirl flow, without separation and unsteadiness, first order turbulence modeling...).

Concerning the definition of the geometrical profile for the nozzle, it must be advised that due to some transversal restrictions in the wind tunnel layout, it was not possible to use a pair of conventional cubic polynomials. Alternatively, other mathematical fittings were explored, keeping in mind the necessity to assure the most significant characteristics of a good designed nozzle: reduction of mean and fluctuating velocity variations, and increase of the flow mean velocity [7].

In this paper, four different contraction profiles (three 4thorder polynomials -FOP- and one logarithmic derivative profile -LDP-) were studied using the Computational Fluid Dynamics 

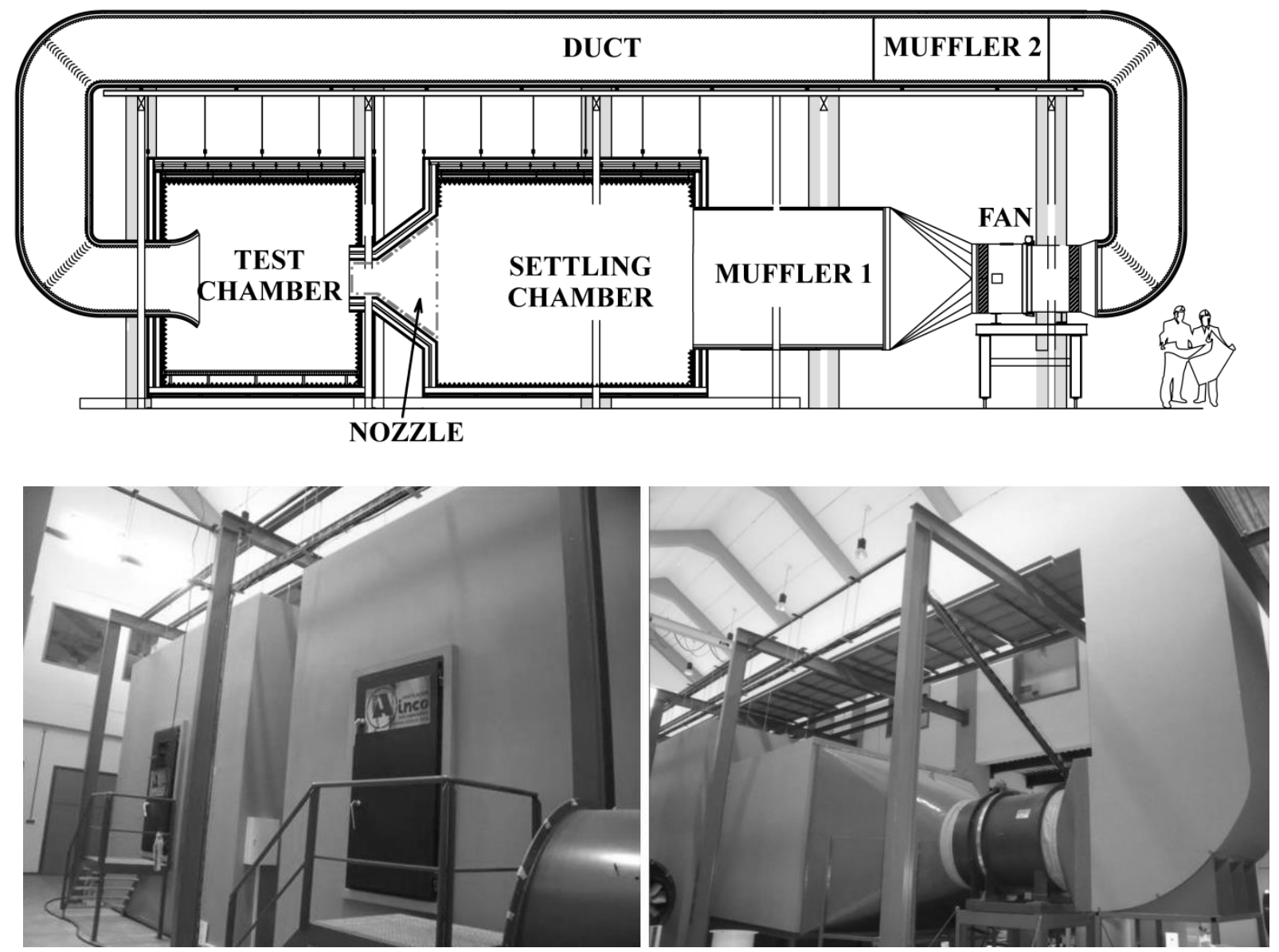

Fig. 1 Wind tunnel facility

(CFD) package ANSYS FLUENT ${ }^{\circledR}$. The design criteria considered for the analysis of the results were the avoidance of separation of the boundary layer, the procurement of a maximum level of exit-flow uniformity, and the obtaining of a minimum turbulence level at the outlet [7]. The numerical results obtained show the benefits of the new profile developed by the authors and justify its final selection for construction in the wind tunnel.

Further validation has been attempted to certificate the goodness of the LDP design. Experimental measurements using pressure taps and hot wire anemometry have revealed the optimal performance of the nozzle in terms of flow uniformity and low turbulence levels (below $0.7 \%$ ); thus concluding that the contraction section developed here was the best option possible for the enhancement of the existing wind tunnel.

\section{DESCRIPTION OF THE WIND TUNNEL}

The wind tunnel of the Fluid Mechanics Group at the University of Oviedo is a closed loop circuit, arranged in a vertical layout, as shown in Fig. 1 . The total length of the tunnel is $24.6 \mathrm{~m}$, with $8.3 \mathrm{~m}$ high and maximum operative velocities in the range of $20 \mathrm{~m} / \mathrm{s}$ for the test section; i.e., a maximum Reynolds number of $7 \cdot 10^{6}$, based on the characteristic entrance length. A full scheme of the facility is also depicted in Fig. 1.

\section{Test chamber}

The test chamber is $4.2 \mathrm{~m}$ long and has a cross sectional area of $4.45 \times 2.80 \mathrm{~m}^{2}$. The dimensions of the test section allow working inside with different equipment and without interference in the air free stream discharged from the nozzle.

\section{Settling chamber}

The settling chamber is the largest chamber of the wind tunnel, with a characteristic cross sectional area of $27.78 \mathrm{~m}^{2}$. Minimum velocities ensure no perturbations of the mean flow and provide accurate flow conditions in the nozzle entrance. The chamber is composed of the following parts: the honey 
comb, the screens and the relaxation duct. The combined effect of these components is to reduce the turbulence coming from the fan, by breaking down larger eddies into smaller ones. The screen holes (see Fig. 2) present a characteristic size of $30 \mathrm{~mm}$, which devises a typical length scale in the range of a tenth of centimeter for the inflow turbulence.

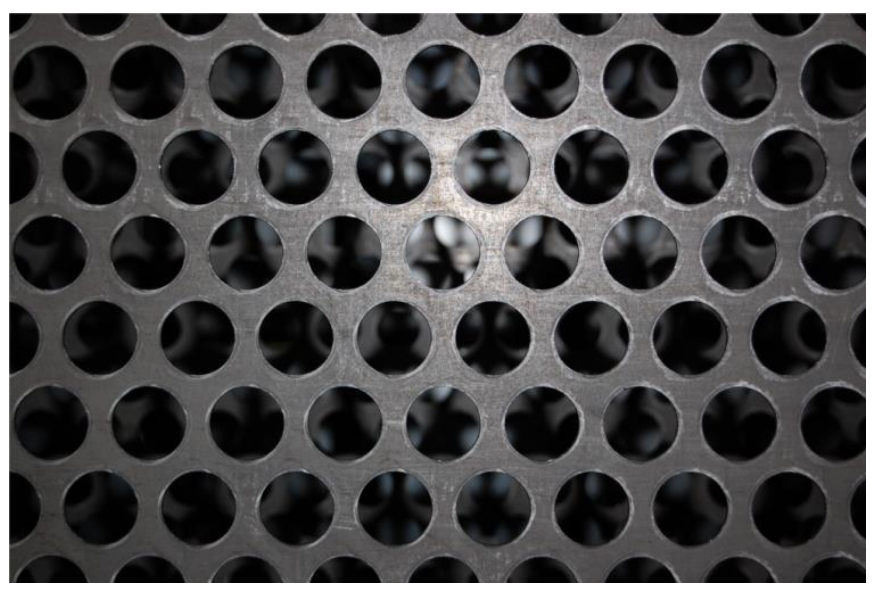

Fig. 2 Screen holes

\section{Mufflers}

Two mufflers, one between the settling chamber and the fan, and another in the longest duct, reduce the noise coming from the fan to the test section. This acoustic isolation makes possible to perform aeroacoustic measurements within the wind tunnel.

\section{Fan}

The fan used is of axial anti-stall type, with the impeller mounted directly on the motor shaft. It provides a variable air flow modifying the pitch of the blades. The $30 \mathrm{~kW}$ driven motor establishes maximum flow rates in the range of $20 \mathrm{~m}^{3} / \mathrm{s}$ (blades are full-opened) and a total-to-static pressure increment of 850 $\mathrm{Pa}$.

\section{Nozzle}

The new nozzle had to be placed between the settling chamber and the test section. Its length was determined by the separation between the chambers $\left(\begin{array}{lll}1.505 & \mathrm{~m}\end{array}\right)$. Different contraction ratios were required for enable diverse experiments, so the nozzle was constructed with the possibility to modify its lateral span. Thus, the distance between the two vertical faces had to be: $2.745 \pm 0.2 \mathrm{~m}$ at the inlet and $1.000 \pm 0.2 \mathrm{~m}$ at the outlet (Fig.3). As a consequence, the contraction ratio (in terms of crossed area) in the nozzle is variable, ranging from 9.5:1 to $6.25: 1$.

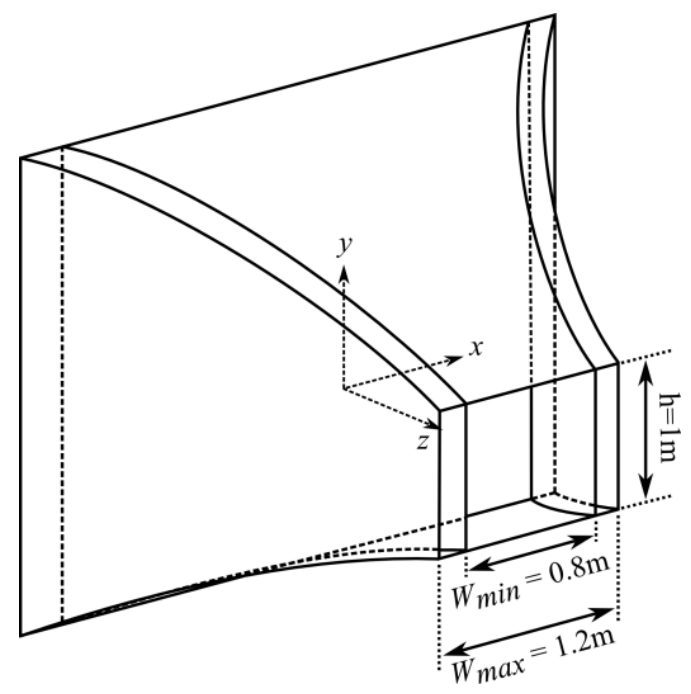

Fig.3 Scheme of the versatile nozzle

\section{NOZZLE CONTOURS STUDIED}

The most extended contraction profiles used for wind tunnels, which have been widely tested and recommended for this type of applications in the literature, are those based in a pair of two cubic polynomials [1-4]. This family of profiles could not be adapted in our case due to some unavoidable geometrical restrictions of the wind tunnel. In particular, the existent structure that connects both chambers limits the space in a way that a two cubic polynomial nozzle cannot be placed in it. To overcome this problem, a fourth order polynomial profile (FOP), given by equation (1), and a logarithmic derivative profile (LDP), given by equation (2), were explored as reasonable alternatives for our design.

$$
y=a_{0}+a_{1} z+a_{2} z^{2}+a_{3} z^{3}+a_{4} z^{4}
$$

$$
y=b_{1}+b_{2} z\left[\ln \left(b_{2} z\right)-b_{3}\right]
$$

The polynomial in equation 1 includes five coefficients (from $a_{0}$ to $a_{4}$ ) that must be fixed according to the following geometrical constraints: Inlet (i) and outlet height (ii), zero acceleration of the air flow at the outlet (iii), zero variation of acceleration of the air flow at the outlet (iv) and a particular inlet curvature (v). Mathematically, these conditions can be expressed as follows:

$$
\begin{aligned}
& y(z=0)=h_{i} \\
& y(z=l)=h_{o} \\
& a(z=l)=0
\end{aligned}
$$




$$
\begin{gathered}
a^{\prime}(z=l)=0 \\
y^{\prime}(z=0)=\operatorname{tg}(\propto)
\end{gathered}
$$

where $h_{i}$ and $h_{o}$ are half inlet and outlet height respectively, $l$ is the nozzle length, $z$ is the longitudinal coordinate in the nozzle, $a$ is the flow acceleration and $\alpha$ is the inlet curvature. The angles chosen for $\alpha$ were 40, 50 and 60 degrees.

Equation 2 was obtained establishing that the nozzle profile follows a logarithmic derivative. This shape was chosen due to its smoothed curvature, with only three parameters defined as:

$$
\begin{gathered}
b_{1}=h_{i} \\
b_{2}=\frac{h_{i}-h_{o}}{l} \\
b_{3}=\ln \left(h_{i}-h_{o}\right)+1
\end{gathered}
$$

which are the aggrupation of the coefficients that adjust the offset and the slope of the logarithmic function. Fig.4.

A representation of the four contraction profiles is shown in

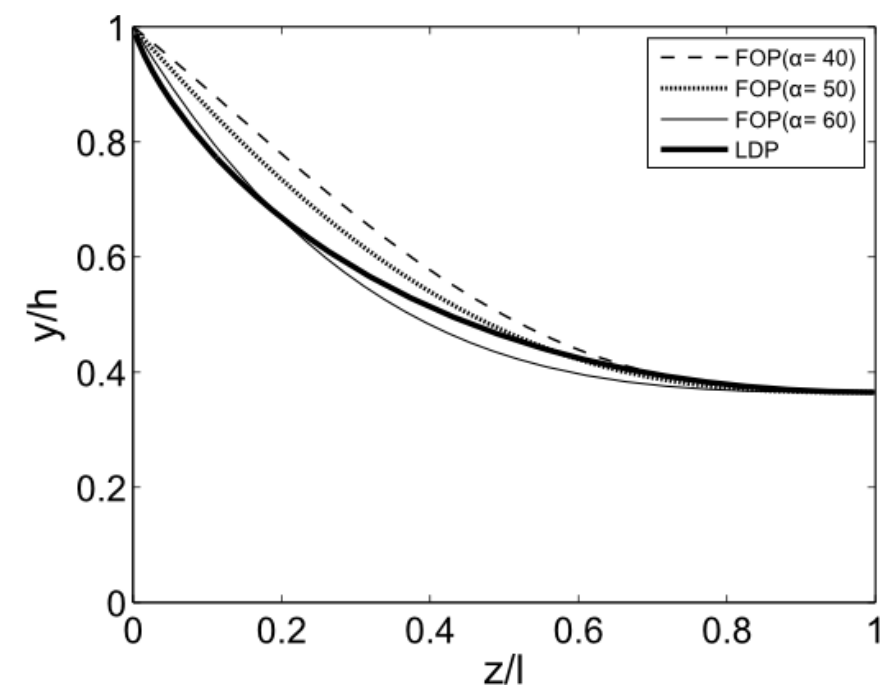

Fig.4 Contraction profiles

In this plot, the $\mathrm{x}$-axis represents the non-dimensional length of the nozzle, whereas the y-axis shows the non-dimensional vertical coordinate. Obviously, the $z$ coordinate increases in the downstream direction. The coordinate system is established with the origin at the center line of the inlet plane. The remaining $x$ coordinate is defined for the spanwise direction.

\section{NUMERICAL METHODOLOGY AND VALIDATION}

\section{Geometry and Mesh}

ANSYS ICEMCFD ${ }^{\circledR}$ meshing software was used for geometry and mesh generation (outlet section $=1 \times 1 \mathrm{~m}^{2}$ ). Due to the symmetry presented, only one quarter of each nozzle was modeled in order to reduce the computational load. Part of the settling duct was also included in the geometry for accuracy purposes, as it is shown in Fig.5.

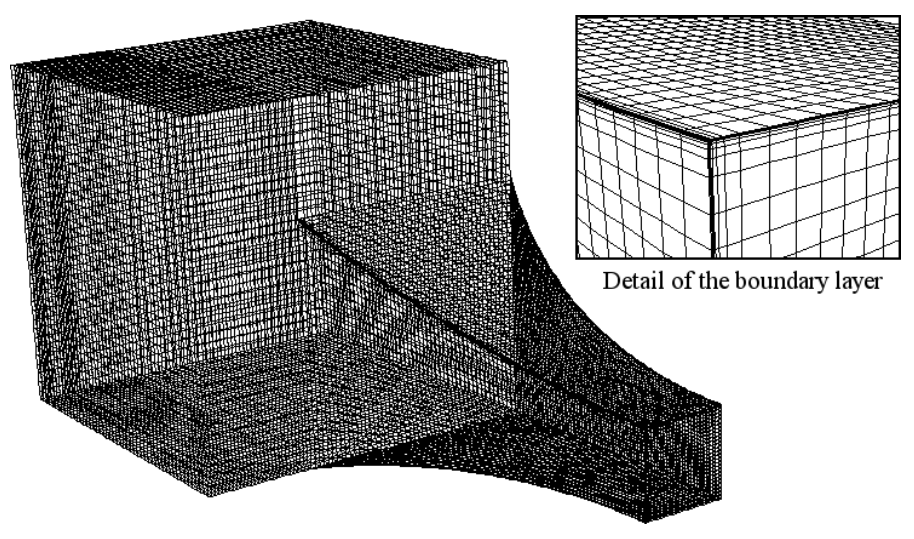

Fig.5 LDP model meshed

Every model was meshed using a structured mesh and avoiding highly skewed elements. A boundary layer grid dependence study, with five different density meshes, was performed using the velocity profiles along the vertical plane of the outlet as control variable. The integral turbulent scales and turbulence intensities at the inlet, measured previously with a hot wire probe, were used for defining a high-density mesh. Further mesh refining was conducted towards the nozzle sidewalls. The comparison of the numerical data obtained for each mesh is illustrated in Fig. 6.

The model with the coarser mesh (100236 cells) is the one that shows the biggest difference in the velocity profile. The velocity starts to drop off when $\mathrm{y} / \mathrm{h}$ is 0.92 , while for the rest of the models it occurs at $\mathrm{y} / \mathrm{h}=0.97$. The results show that refining the mesh from a value of 161112 cells do not provide more accurate values of velocity at the boundary layer. The velocity values are slightly lower near the wall for the 161112 cells model than for the three finest mesh models. For them the results remain invariant. Taking into account this outcomes, the 204120 cells model, the coarser mesh with the best results, was the chosen one for continuing the profile study. The minimum $y^{+}$obtained with this mesh was 0.29 . 


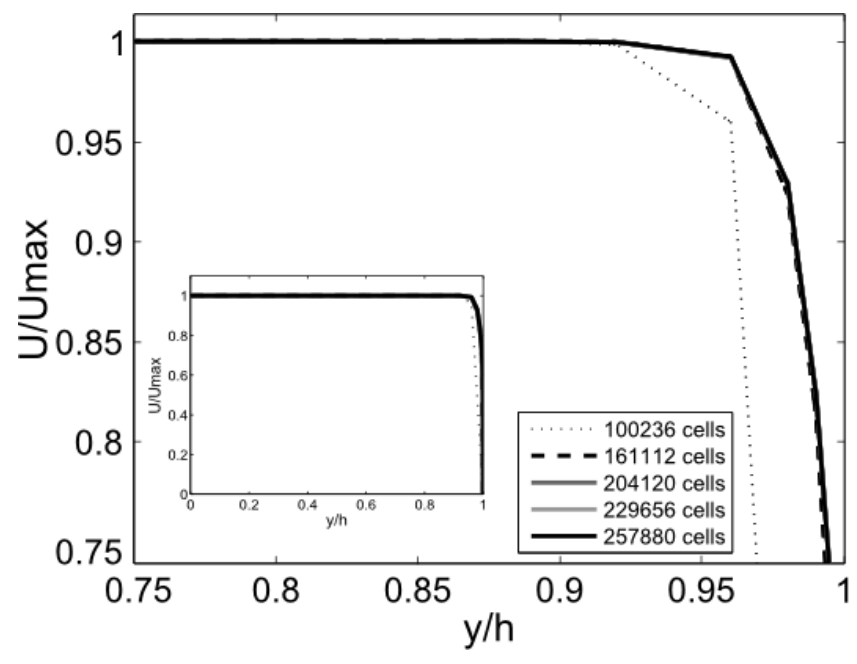

Fig. 6 Numerical comparison of velocity profiles on vertical plane

\section{Boundary conditions and models}

The Computational Fluid Dynamics (CFD) package ANSYS-FLUENT ${ }^{\circledR}$ was used for solving the 3D steady NavierStokes equations in conjunction with the K-epsilon turbulence model and enhanced wall treatment on the structured meshes. This turbulence model was chosen because it obtains the production and dissipation of turbulence and it allows simulate possible pressure gradients leading to boundary layer separations. A SIMPLE pressure-velocity coupling algorithm was selected and second order discretization was applied for the equations to increase the accuracy of the results.

As boundary conditions, it was considered a gauge total pressure of $296.45 \mathrm{~Pa}$ at the inlet with a turbulence intensity of $2.5 \%$ and a turbulent length scale of $0.25 \mathrm{~m}$, both obtained with hot wire measurements at the inlet. It was also imposed a $0 \mathrm{~Pa}$ gauge pressure at the outlet. Both are pressure conditions instead of velocity ones in order not to affect simulation results.

\section{NUMERICAL RESULTS}

The flow uniformity of each model at the outlet was studied and the results obtained are shown in Fig.7. Due to the geometrical resembling of the contraction profiles, it was expected a similar velocity tendency at the outlet of the nozzle. The flow velocity of all models remains greater than $99 \%$ of Umax until it is reached the $96 \%$ of the outlet height. Noting a detail of the velocity profiles in the boundary layer area, some differences can be observed. At this point the LOP profile presents slightly higher velocity values than the polynomial ones, achieving a better flow uniformity.

The velocity contours of the LDP model at the middle plane is depicted in Fig. 8. The flow coming from the settling chamber, with a mean velocity of $4.1 \mathrm{~m} / \mathrm{s}$, is accelerated throughout the nozzle until its way out. At spanwise sections close to the nozzle inlet, the flow velocity increases as it nears the wall. As long as the air advances towards the outlet, this tendency decreases being reversed nearby the outlet.

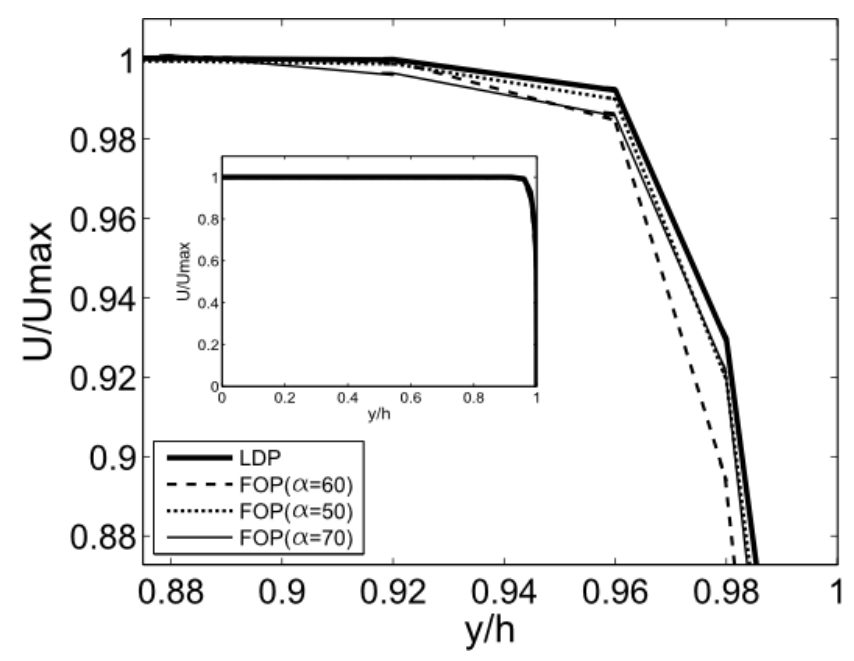

Fig.7 Velocity profiles at the outlet on vertical plane

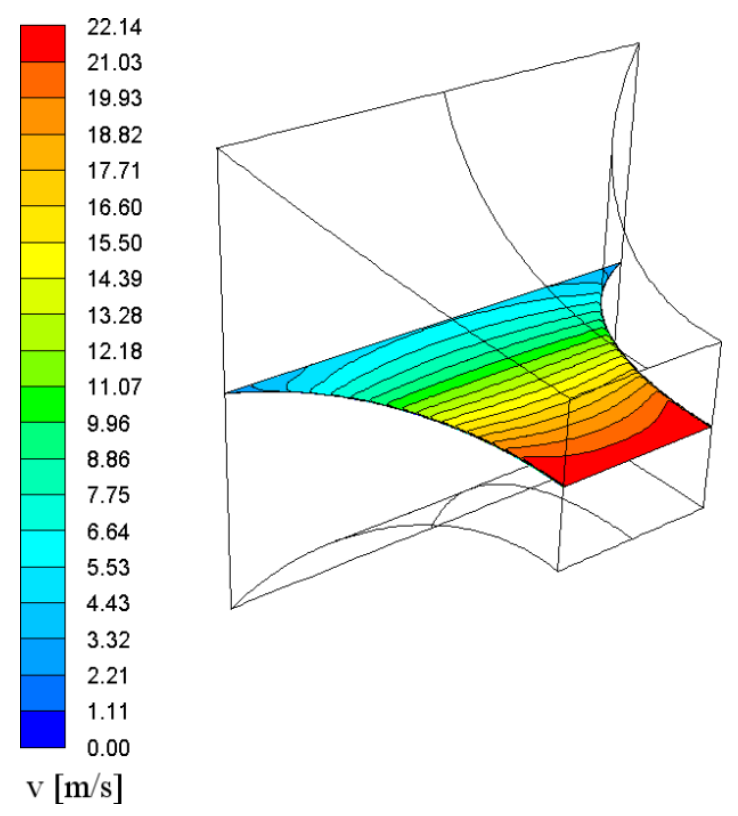

Fig. 8 Velocity contours of LDP on middle plane

As the nozzle structure consists of convex surfaces, the possible separation region spans the entire length of the contraction. For that reason is fundamental that the profile leads the flow avoiding adverse pressure gradients which are the essential condition for the separation. For testing the avoidance of separation at the nozzle walls, the skin friction coefficient was considered (Fig. 9). Simulation results show that the LDP and the FOP $\left(\alpha=50^{\circ}\right)$ do not present separation, indicated by the positive skin friction coefficient values over the wall [8]. However, LDP offers more balanced results without drastic increasing gradients of the coefficient. On FOP $\left(\alpha=60^{\circ}\right)$ and 
FOP $\left(\alpha=40^{\circ}\right)$ there is a region at the wall, near the outlet, that shows a discontinuity of the skin friction coefficient distribution. The fact that the skin friction coefficient at those areas doesn't achieve negative values is justified by the boundary condition imposed at the outlet, a gauge pressure of $0 \mathrm{~Pa}$. Hence it can be conclude that this two profiles (FOP $\left(\alpha=60^{\circ}\right)$ and FOP $\left(\alpha=40^{\circ}\right)$ ) produce the separation of the flow at the boundary layer near the outlet.
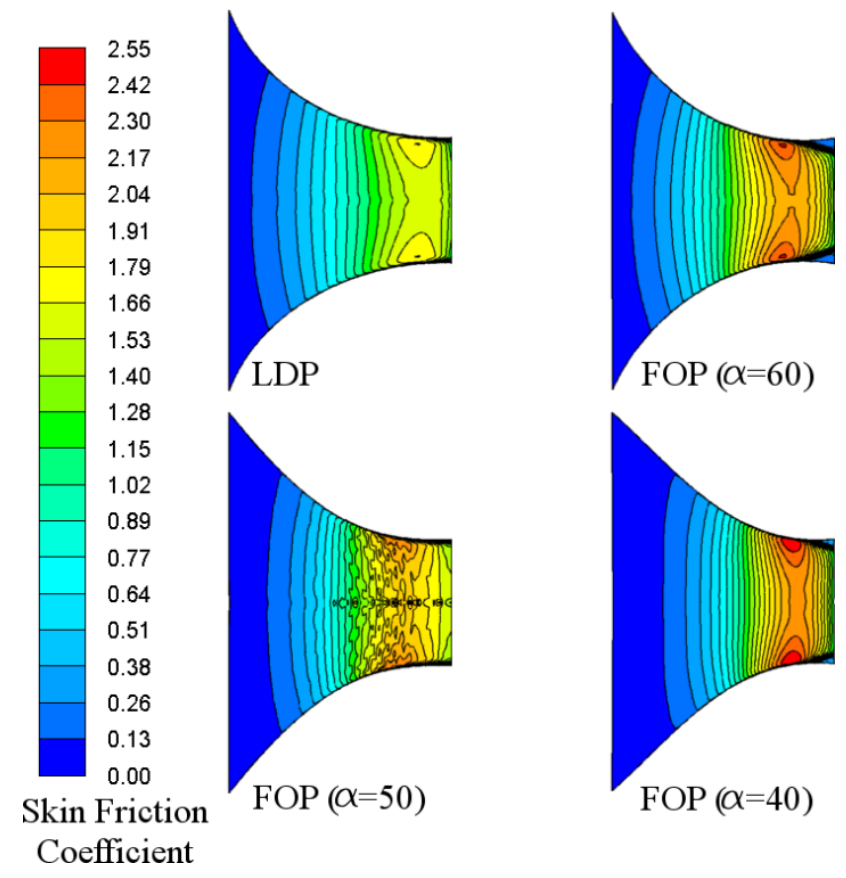

Fig. 9 Skin friction coefficient contours at the nozzle upper wall

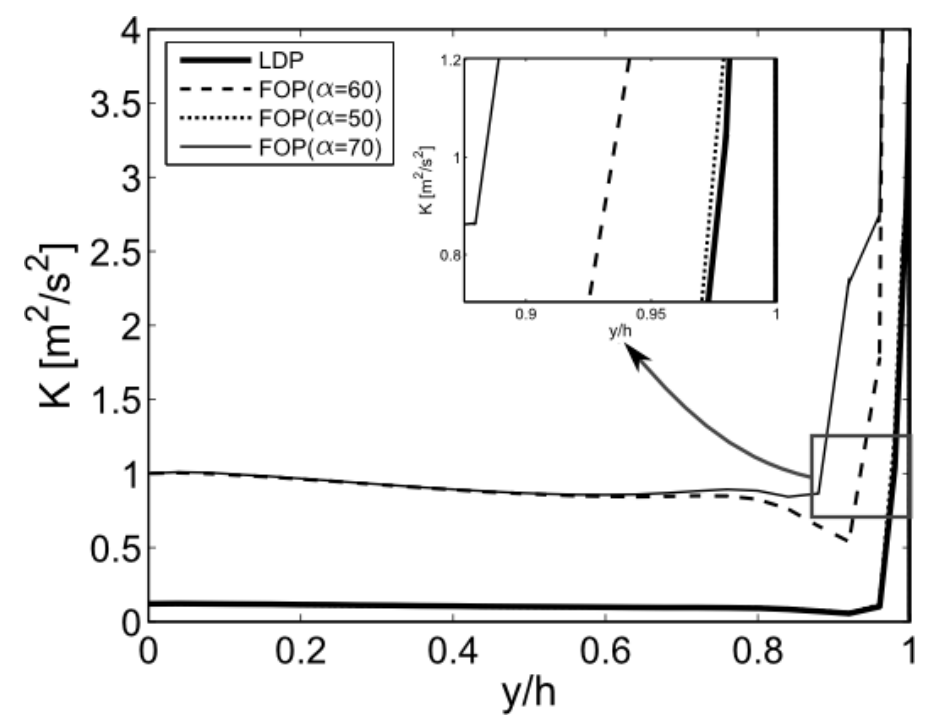

Fig. 10 Turbulent kinetic energy profiles at the outlet on vertical plane

The turbulence generated in the air flow is also an important factor to be considered. It has been analyzed the turbulent kinetic energy profiles at the outlet on vertical plane (Fig. 10). As it was expected, the predicted values for FOP $\left(\alpha=60^{\circ}\right)$ and FOP $\left(\alpha=40^{\circ}\right)$ are much higher due to the separation of the boundary layer. The LDP and the FOP $\left(\alpha=50^{\circ}\right)$ models produce less turbulence at the outlet, reaching almost the same values. The turbulent kinetic energy of both profiles maintains a constant value of $0.1 \mathrm{~m}^{2} / \mathrm{s}^{2}$ until it is reached the $96 \%$ of the outlet. From that point $\mathrm{K}$ increases its value to $3.78 \mathrm{~m}^{2} / \mathrm{s}^{2}$.

As a conclusion, numerical results obtained with the simulation indicate that the logarithmic profile (LOP) and the polynomial profile (FOP $\left(\alpha=50^{\circ}\right)$ ) provide very good results in terms of flow quality for experimental tests. However, the outcomes show that the flow guided by LOP nozzle is slightly better conditioned. Accordingly, this was the shape chosen for building the nozzle.

\section{EXPERIMENTAL SETUP}

Following the numerical results, the LDP was the chosen profile for constructing the wind tunnel contraction (Fig.11).

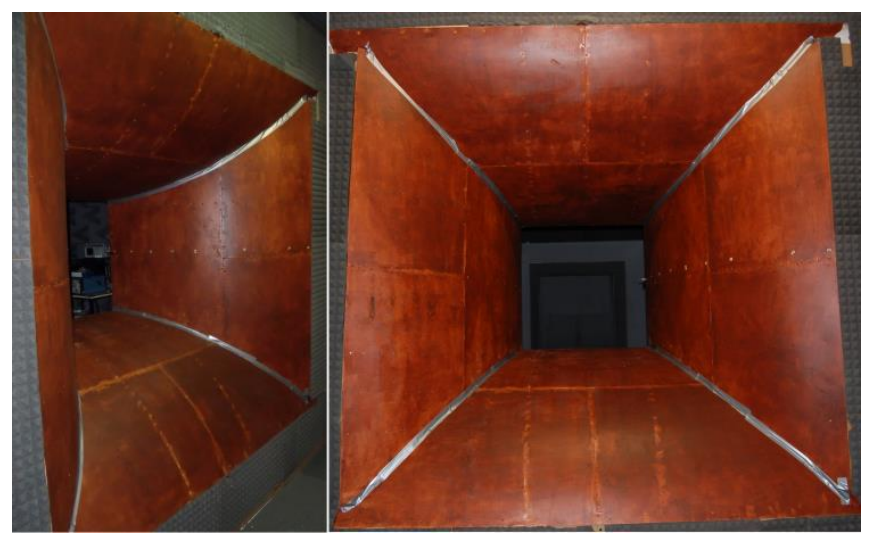

Fig.11 Wind tunnel contraction constructed

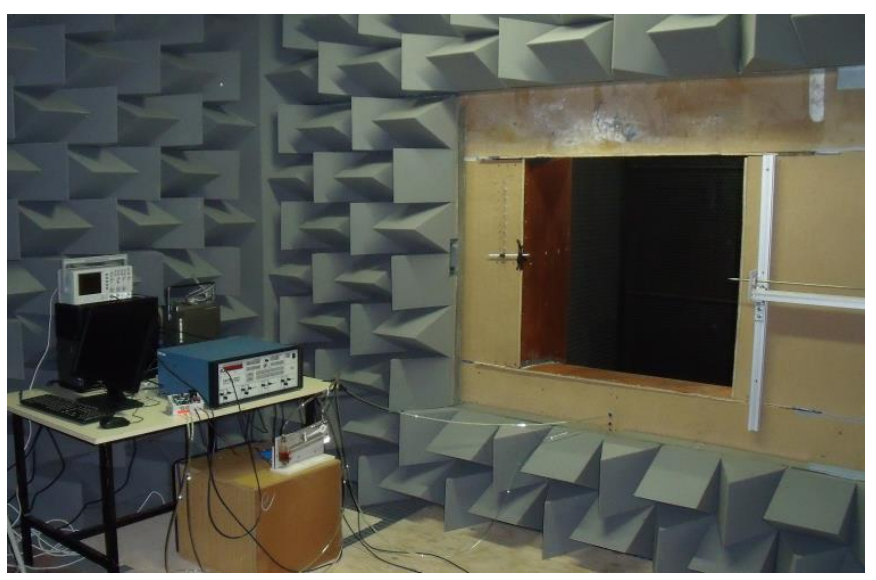

Fig.12 Experimental setup for hot wire measurements 
Ten pressure taps were installed along the middle section of a sidewall, and another two were put at the inlet and the outlet, respectively. These taps, connected to a pressure transducer, provided the wall static pressure distribution and the dynamic pressure at the nozzle outlet.

The velocity distribution and the turbulence intensity at the outlet of the contraction were measured with a home-made constant temperature $\mathrm{X}$-wire probe, connected to a TSI IFA 100.The probe had a cut-off frequency of $64 \mathrm{kHz}$. The IFA 100 outputs where connected to a National Instruments acquisition card that gave the information to a computer. All the aspects of calibration of the probe, capture, record and conversion of measurements were driven by a MATLAB ${ }^{\circledR}$ code, particularly developed for this kind of applications. Note that the authors present a notable background in the use and design of these experimental techniques [9-11]. Fig.12 shows the experimental setup placed in the test section, inside the wind tunnel. The Xprobe was placed at several positions on the center line, between the middle point and the top wall (see Fig. 13 and Fig.14).

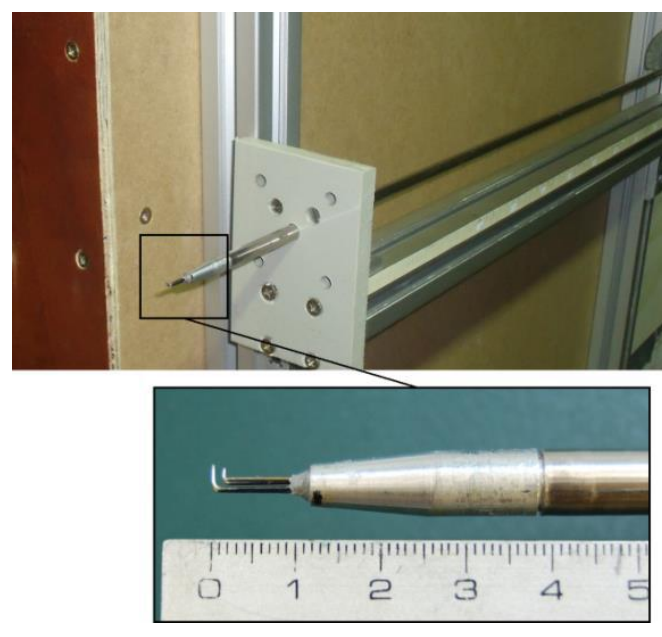

Fig. 13 Assembly and detail of the X-probe

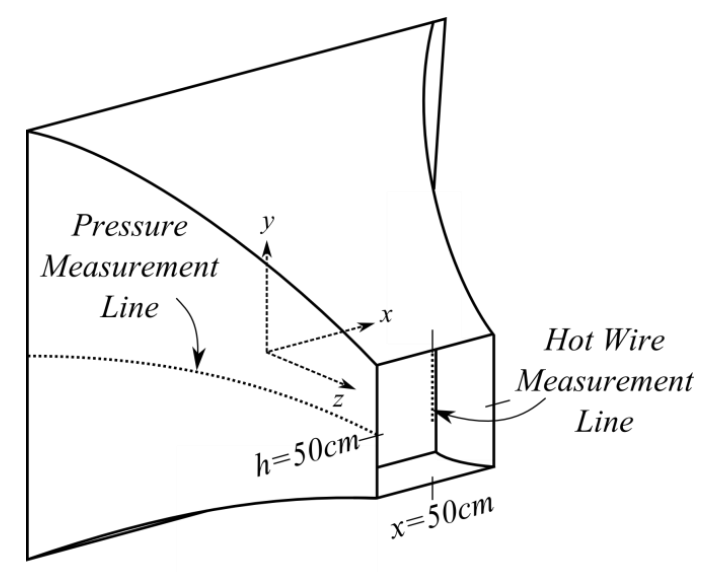

Fig.14 Scheme of measurement lines
Different sampling frequencies at 5, 10, 20 and $50 \mathrm{kHz}$ were tested. The lower frequencies $(5-10 \mathrm{kHz})$ were found not to provide enough accurate data about the turbulence of the flow, while the higher frequencies $(20-50 \mathrm{kHz})$ did not show that problem. It was necessary to find a compromise between the accuracy of the results and the time and space needed for recording the data so, finally, a $20 \mathrm{kHz}$ sampling frequency was chosen.

\section{EXPERIMENTAL RESULTS}

\section{Longitudinal pressure distribution}

The static pressure measured along the middle section of a sidewall was compared with the theoretical static pressure (ideal flow with no losses). The dimensionless parameter $C_{p}$ was used to normalize the data:

$$
C p=\frac{P-P_{o}}{\frac{1}{2} \rho U_{o}^{2}}
$$

where $P$ is the static pressure measured along the wall, $P_{o}$ and $U_{o}$ are the pressure and the mean velocity measured at the center of the outlet, respectively.

A good agreement was found between both distributions all over the curve (see Fig.15). The static pressure at the inlet of the nozzle becomes dynamic pressure at the outlet without significant losses.

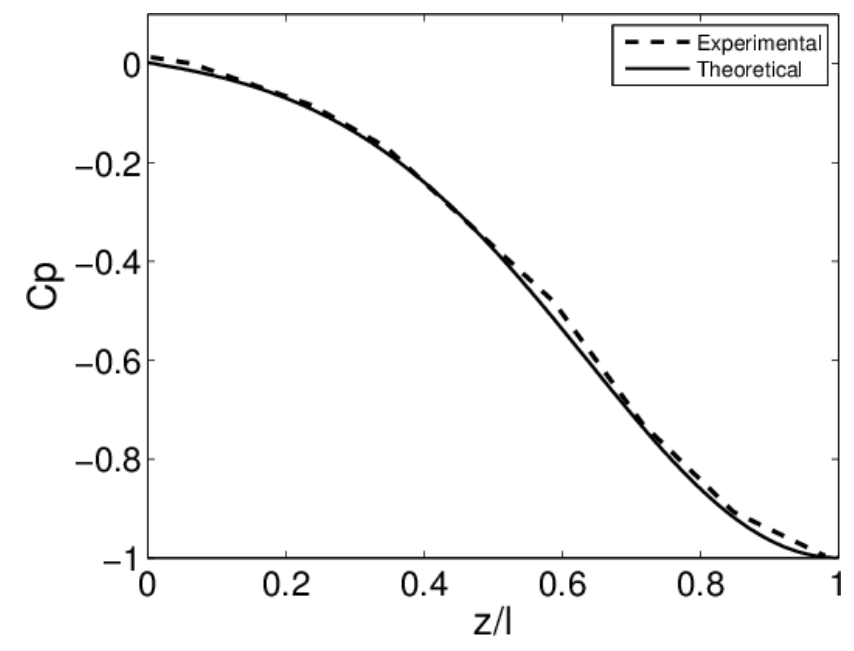

Fig.15 Experimental and theoretical pressure coefficient along a sidewall

\section{Nozzle outlet velocity and turbulence measurements}

A hot wire probe was positioned at the middle line in vertical plane and was traversed from the center of the nozzle outlet to the top wall. This instrument was used to perform 
velocity and turbulence measurements, which provide information about the influence of the walls on the flow.

A first study of the signals recorded showed, as expected, that the reduction of velocity fluctuations was remarkable as the probe approached the center of the nozzle. This fact revealed, at first sight, the significant reduction of the turbulence level throughout the outlet. A fragment of three signals time spectra, recorded at three particular positions, is depicted in Fig. 17. The similarity of the signals at $\mathrm{y} / \mathrm{h}=0.9$ and 0.99 becomes clear; there is no significant variation of the flow velocity, in contrast with the data dispersion near the outlet wall $(\mathrm{y} / \mathrm{h}=0)$.

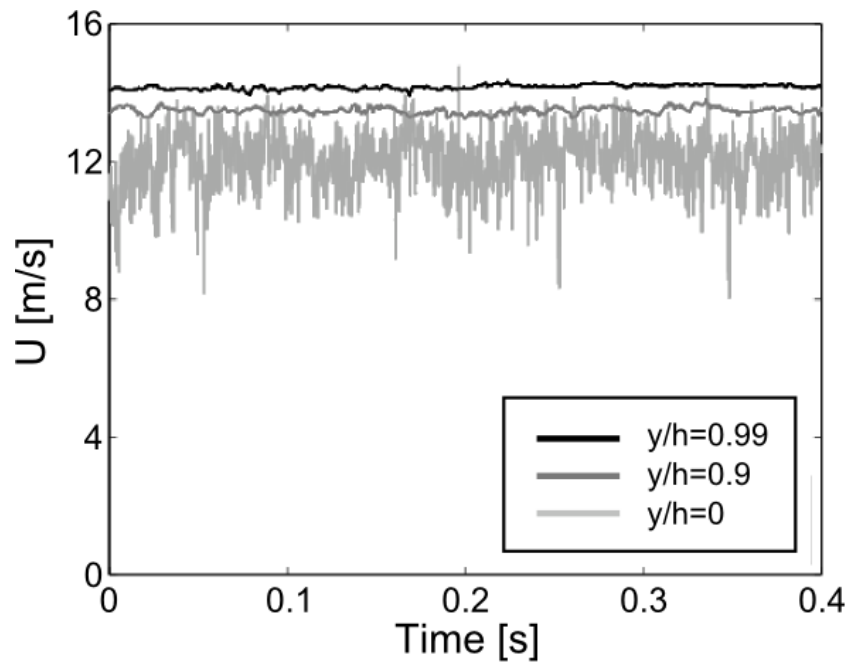

Fig. 16 Time series of signals recorded at $x=50 \mathrm{~cm}$ in vertical plane $(\mathrm{y} / \mathrm{h}=0.5,0.05,0.005)$

The mean velocity values measured at each position and normalized by the maximum velocity are presented in Fig. 17. It is also showed at the same figure the turbulent intensity level, estimated by

$$
T I=\frac{\sqrt{\overline{u^{2 \prime}}}}{\bar{u}} \times 100
$$

where $u$ is the instantaneous velocity in the outlet at the location of the hot wire.

Velocity values remain almost constant from the center of the contraction outlet until $\mathrm{y} / \mathrm{h}=0.01$. The test section flow nonuniformity, based on the difference between the minimum and the maximum nozzle outlet velocity outside the boundary layer [12], was found to be good (2.3\%). The turbulence intensity level estimated is low even at positions near the wall. It is practically constant from the center to $\mathrm{y} / \mathrm{h}=0.1$, where turbulence intensity roughly reaches $0.6 \%$. From $\mathrm{y} / \mathrm{h}=0.025$ until the last measurement position, the mean TI rises up to $1 \%$. The turbulence intensity was also measured at the center of the nozzle inlet. At that position the TI was found to be $2.5 \%$, which indicates that the LPD profile chosen reduces significantly the turbulence level coming from the settling chamber.

The integral length scale of the largest eddies of the flow was determined by

$$
L=\bar{U} \int_{0}^{\infty} A C F(\tau) d \tau ; \quad A C F(\tau)=\frac{\overline{u^{\prime}(t) u^{\prime}(t+\tau)}}{\overline{u^{\prime 2}}}
$$

where the overbar denotes the time-averaging value, and $\tau$ is the time lag that is used to construct the ACF. This formulation assumes that the average eddy size lies through the correlation of two velocity signals (Taylor's hypothesis).
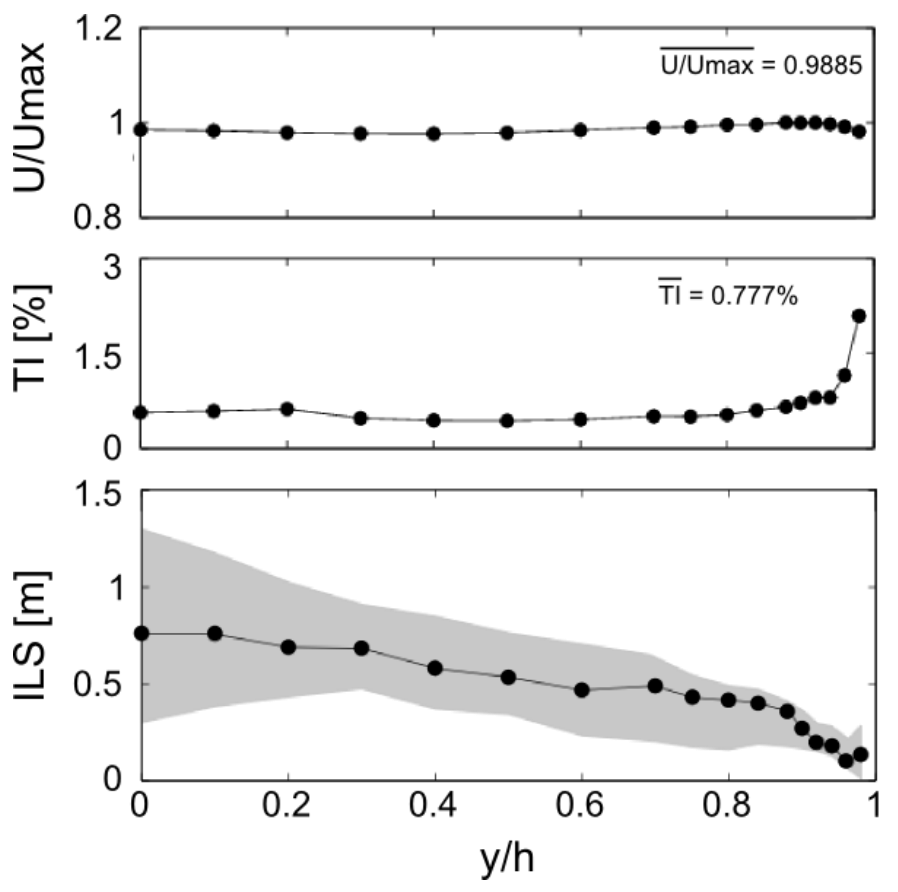

Fig. 17 Normalized wind speed, turbulent intensity and integral length scale measured at $\mathrm{x}=50 \mathrm{~cm}$ in vertical plane

The integral length scale obtained at the nozzle outlet is shown in Fig. 17. The dotted line represents the sizes of the largest eddies obtained as an average of all the data recorded during the measurement campaign, while the grey area shows the dispersion of the ILS values. The magnitude of the uncertainty is due to the inherent randomness of the fluctuations. From the center of the outlet to $\mathrm{y} / \mathrm{h}=0.8$ approximately, the integral lengths scale exhibits a near lineal decay. The diminution of the eddy sizes as the wall is approached is directly related with the increment of the turbulence intensity at that area and its energy dissipation rate.

The variation of the turbulence with the wind speed was also studied. For this survey, the X-probe was fixed at the center of the contraction outlet. Measurements were made with twenty different wind speeds. The results are plotted in Fig.18. 
One can note that the turbulence intensity levels do not differ much. They maintain an approximately constant level of $0.69 \%$.

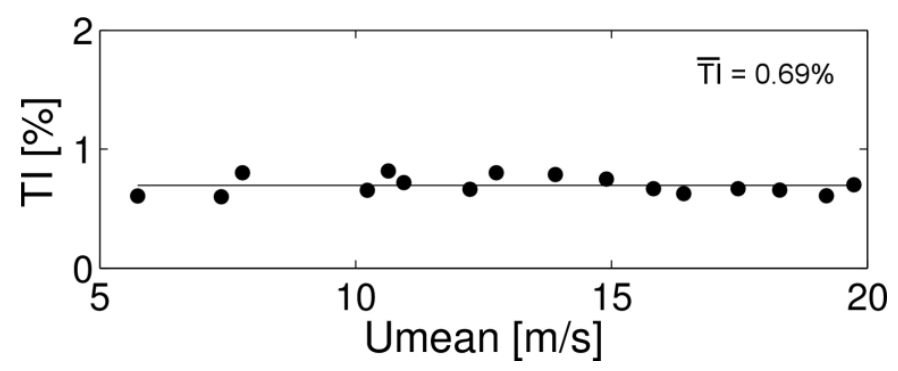

Fig.18 Turbulence intensity level for different wind speeds measured at the center of the nozzle outlet.

\section{Nozzle outlet frequency spectra measurements}

Fig. 19 compares the power spectral density of the free stream region $(\mathrm{y} / \mathrm{h}=0)$ and the viscous region $(\mathrm{y} / \mathrm{h}=0.99)$. The PSD level at the viscous region is higher throughout all the frequency range, related with the turbulence in the boundary layer. Both signals show a decay of the PSD with the frequency, with a broadening of the spectra which means the evolution of the flow searching a fully-developed state. It was verify that both slopes where greater than the $-5 / 3$ exponential decay [13].

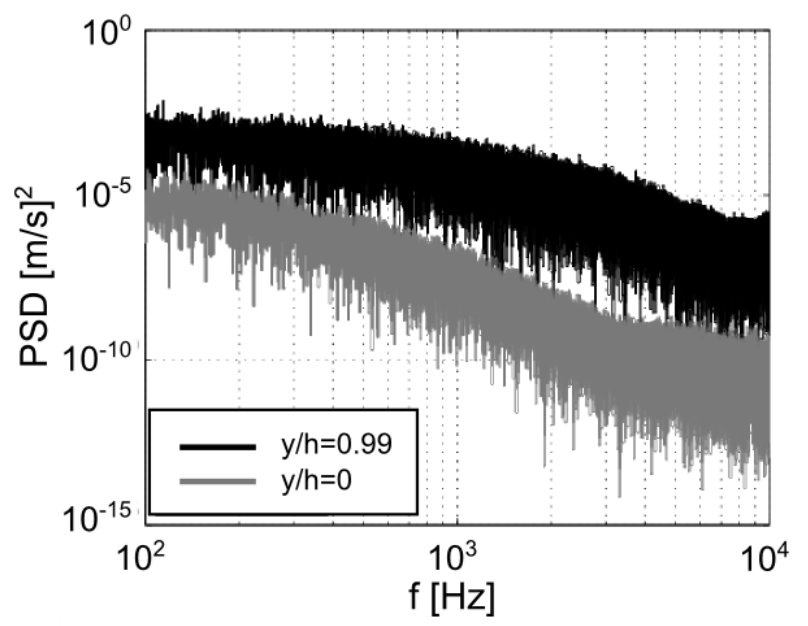

Fig. 19 Power spectral density at the center of the nozzle and near the wall

\section{CONCLUSIONS}

The design, fabrication and characterization of a nozzle to enhance aerodynamic measurements in a low-speed wind tunnel, is presented in this paper. A numerical comparison of the novel design developed by the authors with logarithmic profiles, were carried out. Results showed the benefits of the logarithmic profile (LDP) in terms of flow uniformity, separation of boundary layer and turbulence levels. Moreover, two polynomial profiles (FOP $\left(\alpha=60^{\circ}\right)$ and FOP $\left.\left(\alpha=40^{\circ}\right)\right)$ tended to produce separation of the flow in regions near the outlet.

Experimental measurements with pressure transducers and both, single and dual, hot wire anemometry were accomplished for characterize the nozzle. The pressure coefficient distribution along the sidewall revealed a perfect agreement with the theoretical design, and the velocity and turbulence levels remained low all over the discharge. Turbulence intensity levels for the whole range of velocities available were found to be lower than $0.7 \%$. These outcomes conclude that the novel contraction developed is a good enhancing of the wind tunnel benefits.

\section{ACKNOWLEDGMENTS}

The work reported in this article is funded by the Spanish Ministry of Science and Innovation (MICINN) under the subprogram Predoctoral Research Grants.

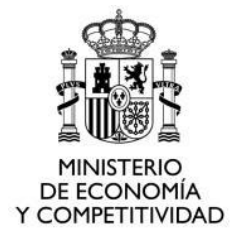

\section{REFERENCES}

[1] Morel T., Comprehensive design of axisymmetric wind tunnel contractions".ASME Journal of Fluid Engineering, 97 (1975) 225-233.

[2] Bell J.H., Mehta R.D., Boundary-layer predictions for small low-speed contractions, AIAA Journal, 27 (1989) 372-374.

[3] Ramaeshan S., Ramaswamy M.A., A rational method to choose optimum design for two dimensional contractions, ASME Journal of Fluid Engineering, 124 (2002) 554-546.

[4] Morel T., Design of two-dimensional wind tunnel contractions, ASME Journal of Fluid Engineering, 99 (1977) 371-378.

[5] Fang F., A design method for contractions with square end sections, ASME Journal of Fluid Engineering, 119 (1997) 454-458.

[6] Fang F., Chen j.C., Hong Y.T., Experimental and analytical evaluation of flow in a square-to-square wind tunnel contraction, Journal of Wind Engineering and Industrial Aerodynamics, 89 (2001) 247 - 262.

[7] Mehta R.D., Bradshaw P., Design rules for small low speed wind tunnels, Aeronautical Journal, 83 (1979) 443-449.

[8] Sargison J.E., Walker1 G.J. and Rossi R., Design and calibration of a wind tunnel with a two dimensional contraction, 15th Australasian Fluid Mechanics Conference 13-17 December (2004) 
[9] Blanco E, Ballesteros R, Santolaria C., Angular range and uncertainty analysis of nonorthogonal crossed hot wire probes. Journal of Fluids Engineering (1998) 120:90-4.

[10] Fernández Oro J.M., Argüelles Díaz K.M., Santolaria Morros C., Blanco Marigorta E., Unsteady flow and wake transport in a low-speed axial fan with inlet guide vanes. Journal of Fluids Engineering, 129- 8 (2007) 1015-1029

[11] Fernández Oro J.M., Argüelles Díaz K.M., Santolaria Morros C., Blanco Marigorta E., On the structure of turbulence in a low-speed axial fan with inlet guide vanes. Experimental Thermal and Fluid Science 32 (2007) 316331

[12] Mathew J., Bahr C., Carroll B., Sheplak M., Cattafesta L., Design, fabrication, and characterization of an anechoic wind tunnel facility. 11th AIAA/CEAS Aeroacoustics conference. (2005).

[13] Davidson P.A., Turbulence: An Introduction for Scientists and Engineers, Oxford University Press, 2004 
This document is a pre-print version of the scientific paper published by Elsevier. It has been released by the authors to fulfill all the publisher requirements established for Article Sharing: https://www.elsevier.com/about/policies/sharing

\section{(9) $\Theta \Theta \Theta$}

(C) 2019. This manuscript version is made available under the Creative Commons Attribution-NonCommercial-NoDerivatives 4.0 International License (CC-BY-NC-ND 4.0 license) http://creativecommons.org/licenses/by-nc-nd/4.0/ 